\title{
P-126: Patternless Vertical Alignment Mode with Infinite Domains for Mobile Displays
}

\author{
Soo In Jo', You-Jin Lee ${ }^{2}$, Sang-Woong Choi ${ }^{1}$, A Ra Yoon ${ }^{2}$, Chang-Jae Yu ${ }^{1,2}$, \\ and Jae-Hoon Kim ${ }^{1,2, *}$ \\ ${ }^{1}$ Dept. of Electronics and Communications Engineering, Hanyang University, Seoul 133-791, Korea \\ ${ }^{2}$ Dept. of Information Display Engineering, Hanyang University, Seoul 133-791, Korea
}

\begin{abstract}
We propose a patternless vertical alignment (VA) mode with infinite domains for mobile displays. Memorizing switching directions of the liquid crystals by a polymerized reactive mesogen on surfaces produced infinite domains and fast response. Also, the transmittance of our patternless VA cell was enhanced by using circular polarizers.
\end{abstract}

\section{Introduction}

High performance liquid crystal displays (LCDs) with wide viewing angle and fast response time characteristics are widely used in small size LCD application such as mobile phone due to its high resolution characteristics. Various approaches have been taken including rubbing of polymer films which is most popular and conventional process. However, a narrow and nonuniform viewing angular characteristic limits the display performance which originated from the fact that all the LC molecules are aligned unidirectionally over the whole panel area. To overcome this problem, many kinds of LC modes such as in-plane switching (IPS), fringe-field switching (FFS), multi-domain vertical alignment (MVA), and patterned vertical alignment (PVA) have been developed for wide viewing angle characteristics using multi-domain alignment of LC molecules [1-10]. However, additional process such as electrode etching, precise assembly between top and bottom substrates and passivation layers are needed. Also actually they do not obtain the real omnidirectional LC alignment but limited numbers of LC domains. Therefore, viewing angle characteristics are not uniform according to the point of the viewer.

Recently, azimuthally continuous nematic domain (ACD) [11] was suggested to generate the omnidirectional LC direction by using slits and the circularly shaped electrodes. However, slit patterns have to be placed in both substrates and they cause the additional fabrication process and cost.

In this paper, we propose a patternless vertical alignment (VA) mode with infinite domains for mobile displays. Memorizing switching directions of the liquid crystals by a polymerized reactive mesogen on surfaces produced infinite domains and fast response. Also, the transmittance of our patternless VA cell was enhanced by using circular polarizers.

\section{Experiments}

Figure 1 shows the electrode configurations of the conventional and proposed structure. In the case of the conventional structures, circularly shaped slit region at the common electrode is essential to generate the obliquely induced electric field between top and bottom substrates. The LC directors which are vertically aligned at initial state fall down to the substrate along the electric field which are induced obliquely between top and bottom substrates and generate the spiral defect structure $(\mathrm{m}=+1)$ under the crossed polarizer in each square shaped electrode at the pixel electrode. Therefore, three spiral defects are generated uniformly in one pixel by using conventional electrode structure. To simplify the fabrication process and reduce the manufacturing cost, it is undisputable to remove the circular shaped slit and adopt the nonpatterned ITO glass substrate as a common electrode. However, if the circular shaped slits were removed, LC directors are unstable with weakly defined field direction, cannot generate the uniform spiral textures and thus cause the slow reorientation process when the electric field induced. Therefore, we propose the patternless VA mode with electrode structure which can generate the uniform spiral textures just as the conventional structure without additional electrode patterning in the common electrode area. Pixel structure is designed like delta structures and connection line between the square pixels can generate the precise oblique field without slit (Fig. 1(b)). Therefore, in patternless VA mode, non-patterned ITO glass substrate is applied as a common electrode and proposed structure is used as a pixel electrode.

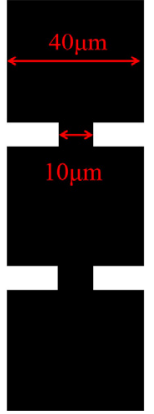

Pixel Electrode

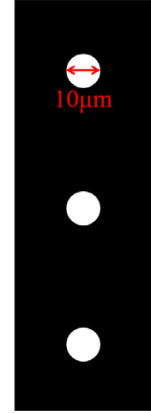

Common Electrode

a) Conventional structure

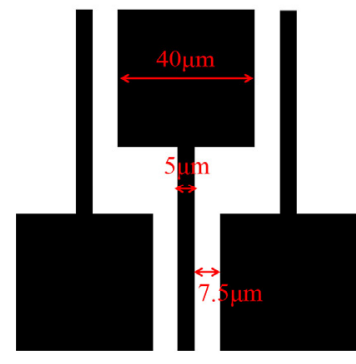

Pixel Electrode
Figure 1. Electrode configurations of the conventional and proposed LCD modes.

To fabricated patternless VA mode LC sample, the mixtures of the vertical alignment material AL1H659 (from JSR) and RM (from Merck) with proper weight ratio were spin coated on both pixel and common ITO substrates. The cell gap was maintained about $3.0 \mu \mathrm{m}$ with glass spacer and the cell was filled with LC (MLC-6608, $\Delta \varepsilon=-4.2$, Merck). Without applying voltage, the LC molecules were aligned vertically due to vertical alignment material and the RM monomers were distributed randomly in the alignment layer. When the applied 
voltage is larger than threshold voltage, the LC molecules fell down with omnidirectionally, and the RM monomers aligned along the LC molecules. After a few times later till the LC directors are stabilized with reorientation process, the cell was exposed to the UV light, and then the RM monomers were polymerized within the alignment layer with certain polar and azimuthal direction along the LC molecules which is controlled by the strength of applied voltage.

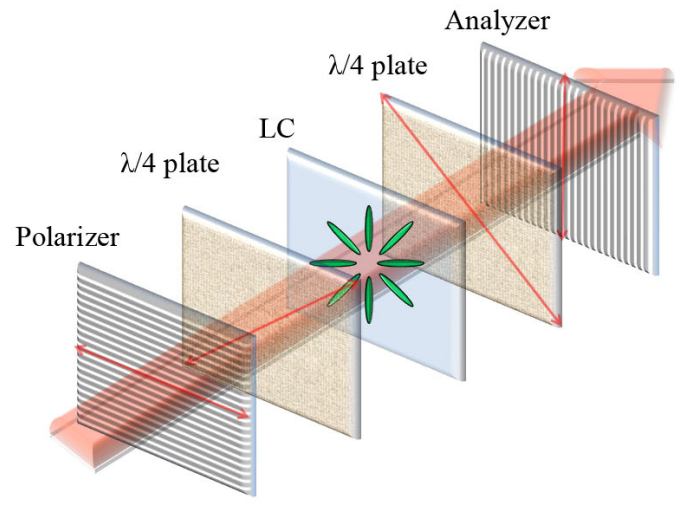

(a) Optical setup for patternless VA mode

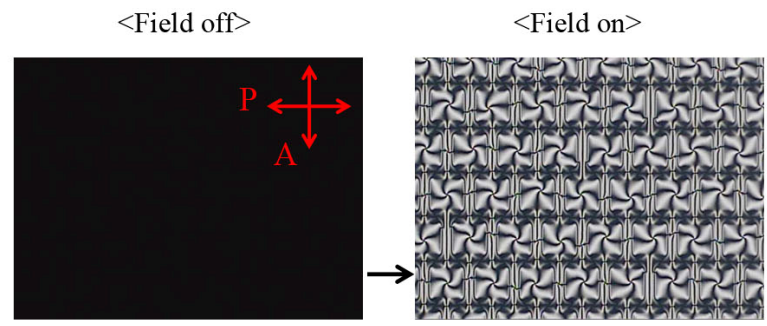

(b) Without $\lambda / 4$ plates

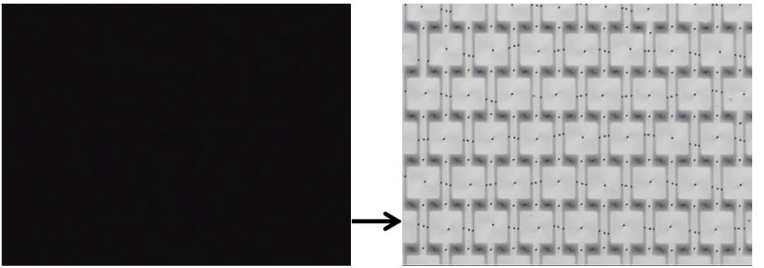

(c) With $\lambda / 4$ plates

Figure 2. The schematic diagram of patternless VA mode: (a) optical setup (the arrows depict the optic axes of each optical component). Textures of the sample under the crossed polarizers (b) without $\lambda / 4$ plates and (c) with $\lambda / 4$ plates.

\section{Result and Discussion}

Figure 2 shows the schematic diagrams and the textures of the proposed patternless VA mode. Our proposed patternless VA mode consists of two crossed polarizers, two $\lambda / 4$ plates and
LC cells. Two $\lambda / 4$ plates are used at the front and back side of the LC sample to increase the transmittance characteristics and their optic axes are $45^{\circ},-45^{\circ}$ with respect to the optic axis of the polarizer, respectively (Fig. 2(a)). And the LC molecules have the spiral structure, as shown in Fig. 2(b), with applied voltage. This indicates that the LC molecules start to fall down to the substrate at the edge of the electrode when the electric field is applied to the sample and propagate to the center of the square shaped electrode in every pixel to stabilize the free energy. And then, after adopting the $\lambda / 4$ plates, those spiral textures are disappeared and the transmittance is increased (Fig. 2(c))

Let us consider the transmittance of our patternless VA mode when the $\lambda / 4$ plates are added to the sample. Using Jones matrix representation, we can simply identify the polarization state of the light at each step after passing through the optical components. The input light is linearly polarized light which optical axis is parallel to the $x$-axis. And optics axes of the $\lambda / 4$ plates are $\pi / 4,-\pi / 4$ to the $x$-axis. The $\lambda / 4$ plate can change the linearly polarized light after the analyzer changes to circularly polarized light and it can remove the defect under crossed polarizers.

$E_{\text {out }}=\left(\begin{array}{ll}0 & 0 \\ 0 & 1\end{array}\right)\left(\begin{array}{rr}\cos \left(-\frac{1}{4} \pi\right) & -\sin \left(-\frac{1}{4} \pi\right) \\ \sin \left(-\frac{1}{4} \pi\right) & \cos \left(-\frac{1}{4} \pi\right)\end{array}\right)\left(\begin{array}{cc}e^{-i \frac{\pi}{4}} & 0 \\ 0 & e^{i \frac{\pi}{4}}\end{array}\right)$

$$
\left(\begin{array}{rr}
\cos \left(-\frac{1}{4} \pi\right) & \sin \left(-\frac{1}{4} \pi\right) \\
-\sin \left(-\frac{1}{4} \pi\right) & \cos \left(-\frac{1}{4} \pi\right)
\end{array}\right)\left(\begin{array}{rr}
\cos \theta & -\sin \theta \\
\sin \theta & \cos \theta
\end{array}\right)
$$$$
\left(\begin{array}{cc}
e^{-i \frac{\pi}{2}} & 0 \\
0 & e^{i \frac{\pi}{2}}
\end{array}\right)\left(\begin{array}{rr}
\cos \theta & \sin \theta \\
-\sin \theta & \cos \theta
\end{array}\right)\left(\begin{array}{rr}
\cos \left(\frac{1}{4} \pi\right) & -\sin \left(\frac{1}{4} \pi\right) \\
\sin \left(\frac{1}{4} \pi\right) & \cos \left(\frac{1}{4} \pi\right)
\end{array}\right)
$$$$
\left(\begin{array}{cc}
e^{-i \frac{\pi}{4}} & 0 \\
0 & e^{i \frac{\pi}{4}}
\end{array}\right)\left(\begin{array}{rr}
\cos \left(\frac{1}{4} \pi\right) & \sin \left(\frac{1}{4} \pi\right) \\
-\sin \left(\frac{1}{4} \pi\right) & \cos \left(\frac{1}{4} \pi\right)
\end{array}\right) \frac{1}{\sqrt{2}}\left(\begin{array}{l}
1 \\
0
\end{array}\right)
$$

$$
\begin{aligned}
T & =\left(\frac{1}{\sqrt{2}}\right)^{2}(\sin (2 \theta)+i \cos (2 \theta))(\sin (2 \theta)-i \cos (2 \theta)) \\
& =\frac{1}{2}
\end{aligned}
$$

After some calculations, we can find the polarization state of the light which is passing through the proposed LC sample. Initially, linearly polarized light parallel to the $\mathrm{x}$-axis is changed to the circularly polarized light due to the $\lambda / 4$ plate, and then LC sample change the handness of circularly polarized light because the optical retardation of the LC sample is $\lambda / 2$. Finally, the second $\lambda / 4$ plate can change the polarization state into linearly polarized light which has parallel direction with y-axis. Therefore, amount of the light does not get lost after passing through the sample. The transmittance $(\mathrm{T})$ is always same regardless of the azimuthal angle $(\theta)$ of LC molecules. Therefore, spiral defects which deteriorate the transmittance characteristics are considered to disappear with microscopic textures. 


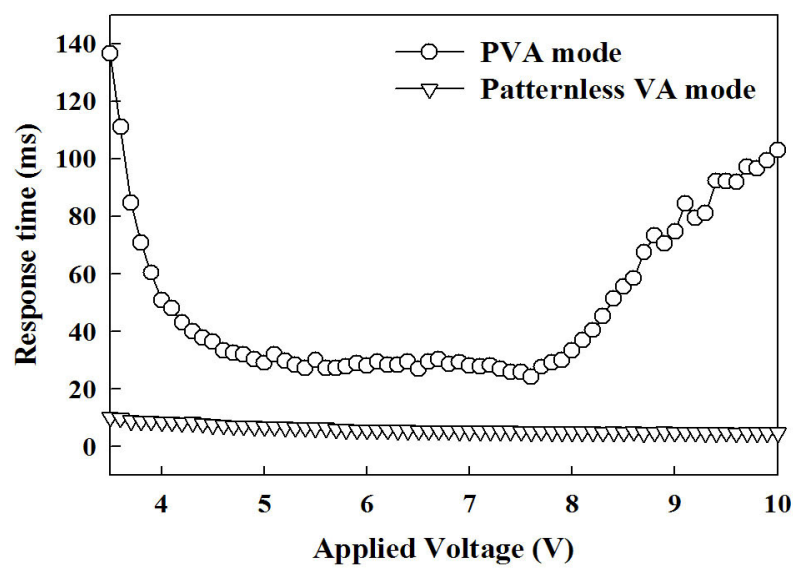

(a) Voltage-Response time

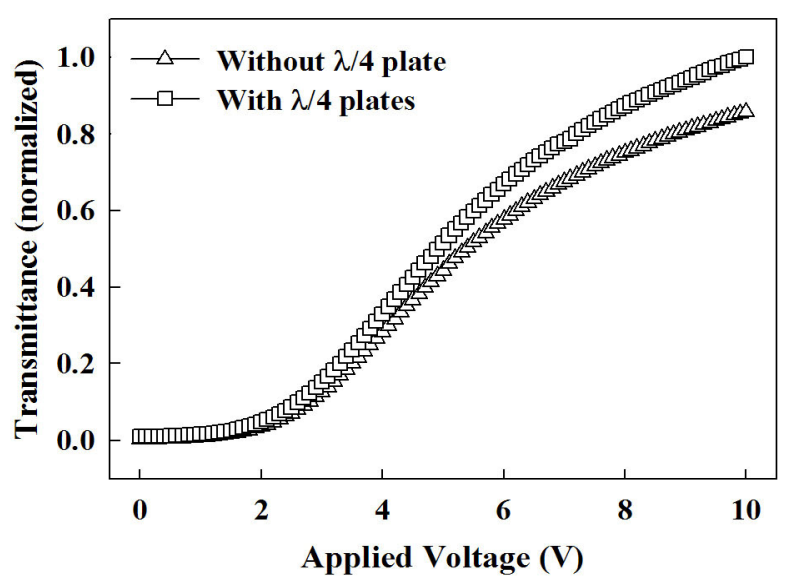

(b) Voltage-Transmittance

Figure 3. Electro-optical characteristics of (a) voltage-response time curves for our patternless VA and the PVA modes and (b) voltage-transmittance curves of our patternless VA mode.

Figure 3(a) shows the response time characteristics as a function of the applied voltage for conventional PVA mode and proposed patternless VA mode. In conventional PVA, the response time was over $100 \mathrm{~ms}$ at low $(\sim 4 \mathrm{~V})$ and high $(\sim 10 \mathrm{~V})$ voltage region due to the propagation of the $\mathrm{LC}$ molecules and slow reorientation process between the slit regions. However, similar to SC-PVA mode [12], the response time of the patternless VA mode was only $5.4 \mathrm{~ms}$ at the $7 \mathrm{~V}$ due to memorized falling direction of LC molecule by polymerized RM within alignment layers at the UV curing process. Figure 3(b) shows the transmittance characteristics with $\lambda / 4$ plates. Transmittance is increased about $17 \%$ due to vanishing of the spiral shaped defect by changing the optical state of the light by $\lambda / 4$ plates.

\section{Conclusion}

We proposed a patternless VA mode with omnidirectional domains of LC molecules. The omnidirectionally distributed LC directors are able to realize the wide viewing angle characteristics and the higher transmittance by adopting the optical modification with $\lambda / 4$ plates. Memorized switching directions of LC molecules by a directionally polymerized RMs on the alignment layers make possible the fast response time without any reorientation process of LC molecules when switching. With those simple process and high efficiency, proposed patternless VA mode could be applicable to the mobile display devices.

\section{Acknowledgements}

This research was supported by Samsung Electronics, LCD R\&D Center.

\section{References}

[1] M. Oh-e, K. Kondo, "Electro-optical characteristics and switching behavior of the in-plane switching mode," Appl. Phys. Lett. 67, 3895 (1995).

[2] S. H. Lee, S. L. Lee, and H. Y. Kim, "Electro-optic characteristics and switching principle of a nematic liquid crystal cell controlled by fringe-field switching," Appl. Phys. Lett. 73, 2881-2883 (1998).

[3] A. Takeda, S. Kataoka, T. Sasaki, H. Chida, H. Tsuda, K. Ohmuro, T. Sasabayashi, Y.Koike, and K. Okamoto, “A super-high-imagequality multi-domain vertical alignment LCD by new rubbing-less technology," SID'98, 1077 (1998).

[4] I. H. Yu, I. S. Song, J. Y. Lee, and S. H. Lee, “' Intensifying the density of a horizontal electric field to improve light efficiency in a fringe-field switching liquid crystal display," J. Phys. D 39, 2367 (2006).

[5] K. H. Kim, K. Lee, S. B. Park, J. K. Song, and J. H. Souk, "Domain divided vertical alignment mode with optimized fringe field effect," The $18^{\text {th }}$ IDRC, 383 (1998).

[6] K. H. Kim, J. J. Ryu, S. B. Park, J. K Song, B.W. Lee, J. S. Byun, and J. H. Souk, "Current status and advance in PVA technology for high performance LCD monitor application," The $1^{\text {st }} I M I D, 58$ (2001).

[7] Y.-J. Lee, T.-H. Lee, J.-W. Jung, H.-R. Kim, Y. Choi, S.-G. Kang, Y.-C. Yang, S. Shin, and J.-H. Kim, "Transflective liquid crystal display with single cell gap in patterned vertically aligned mode," Jpn. J. Appl. Phys. 45, 7827 (2006).

[8] C.-S. Lee, H.-J. Yoon, S.-I. Yoon, and S.-H. Yoon, "Improved PVA mode with high transmittance and aperture ratio," SID’05, 982 (2005).

[9] C. Heinmjiller, G. Haas, and P. M. KnollSf, "Design of in-planecompensation foils for viewing angle enhancement," SID'99, 90 (1999).

[10]R. A. Soref, "Field effects in nematic liquid crystals obtained with interdigital electrodes," J. Appl. Phys. 45, 5466 (1974)

[11]K. S. Bae, J. S. Gwag, I.-Y. Han, Y.-J. Lee, Y. Choi, J.-S. Choi, and J.-H. Kim, "Azimuthally continuous nematic domain mode using electrode structure with circular slit," SID'08, 1951 (2008).

[12]Y.-J. Lee, Y.-K. Kim, S. I. Jo, J. S. Gwag, C.-J. Yu, and J.-H. Kim, "Surface-controlled patterned vertical alignment mode with reactive mesogen," Opt. Exp. 17, 10298 (2009). 